CERN-PPE/95-111

$19 / 06 / 1995$

\title{
Particle Identification Performance of a Straw Transition Radiation Tracker Prototype
}

T. Åkesson ${ }^{1)}$, H. Carling ${ }^{1)}$, B. Dolgoshein ${ }^{2}$, C. W. Fabjan ${ }^{3)}$, P. Farthouat ${ }^{3)}$, D. Froidevaux ${ }^{3)}$, W. Funk ${ }^{3)}$, S. Furletov ${ }^{2}$, I. Gavrilenko ${ }^{3)}$, M. Holder ${ }^{4)}$, V. G. Ivochkin ${ }^{5)}$, S. Jagielski ${ }^{6)}$, O. Kondratiev ${ }^{2}$, S. Konovalov ${ }^{7}$, A. Konstantinov ${ }^{2)}$, M. Kopitin ${ }^{7)}$, P. Lichard ${ }^{3)}$, S. Muraviev ${ }^{7)}$, A. V. Nadtochi ${ }^{5}$, P. Nevski ${ }^{3}$, P. R. Norton ${ }^{8)}$, V. Peshekhonov ${ }^{9)}$, V. A. Polychronakos ${ }^{10)}$, M. Potekhin ${ }^{2) 14)}$,

V. Radeka ${ }^{10)}$, R. Richter ${ }^{11)}$, A. Romaniouk ${ }^{2}$, D. H. Saxon ${ }^{12)}$, V. A. Schegelsky ${ }^{5}$, J. T. Shank ${ }^{13)}$, A. Shmeleva ${ }^{7)}$, S. Smirnov ${ }^{2)}$, V. Sosnovtsev ${ }^{2)}$, E. M. Spiridenkov ${ }^{5)}$, M. Stavrianakou ${ }^{12)}$, D. Stephani ${ }^{10)}$, V. Tcherniatin ${ }^{2)}$, L. Vassilieva ${ }^{7)}$, J. S. Whitaker ${ }^{13)}$, N. Zaganidis ${ }^{3)}$

\begin{abstract}
An 864 channel prototype of an integrated straw tracker and transition radiation detector for tracking and electron identification has been tested with and without magnetic field at the CERN SPS. The rejection against hadrons and converted photons has been measured and the dependence of the rejection power on detector parameters has been investigated. Tracking and hadron rejection were also studied in a high multiplicity environment. The results are compared with Monte-Carlo simulations. Wherever possible, conclusions are drawn concerning the performance of a full-scale detector at the future Large Hadron Collider.
\end{abstract}

Submitted to Nuclear Instruments and Methods

\footnotetext{
1) University of Lund, Sweden

2) Moscow Physical Engineering Institute, Moscow, Russia

3) CERN, Geneva, Switzerland

4) Siegen University, Germany

5) Nuclear Physics Institute, St. Petersbourg, Russia

6) Institute of Nuclear Physics, and Academy of Mining and Metallurgy, Krakow, Poland

7) P. N. Lebedev Institute of Physics, Moscow, Russia

8) Rutherford Appleton Laboratory, Didcot, UK

9) JINR(Dubna), Russia

10) Brookhaven National Laboratory, Upton, NY, USA

11) MPI, Munich, Germany

12) Glasgow University, UK

13) Boston University, Boston, Mass.,USA

14) now at Columbia University, New York, NY,USA
} 
One of the most difficult tasks for an experiment at the future high luminosity Large Hadron Collider (LHC) at CERN will be the reconstruction of charged particle trajectories and their identification, before most of them are absorbed in the calorimeters. The main difficulties lie in the high particle densities per bunch crossing, the short time intervals between bunch crossings and the large radiation doses that the detectors have to survive.

Moreover, in view of the fact that one of the most practical ways to observe the decays of massive new particles, among all the strong interaction products, is to search for their leptonic decays, lepton identification will be of great importance. In contrast to the existing hadron colliders, where the isolated electron to jet ratio at transverse momenta of approximately $40 \mathrm{GeV}$ is about $10^{-3}$, this ratio is expected to be much lower at the $\mathrm{LHC}$, of the order of $10^{-5}$. The expected calorimeter rejection of hadronic jets, while retaining a high efficiency for electrons, is of the order of $10^{3}$. The inner detector of an LHC experiment must therefore provide an additional rejection of about $10^{3}$ in order to reduce the jet backgrounds to less than $10 \%$ of the inclusive isolated electron signal, and, at the same time, be capable of stable operation in the harsh environment of the high luminosity collider.

One approach to a detector comprising the features of tracking and particle identification was first proposed at the SSC instrumentation workshop in Berkeley 1987 [1], further developed by the High Luminosity Working group at CERN [2] and has been an R\&D project $[3,4]$ within the DRDC framework at CERN over the past five years.

The development of this instrument progresses through construction and tests of prototype detectors and detailed computer simulations. This paper presents the test beam results from the second generation of these prototypes and extrapolates the test beam measurements, using computer simulations, to the performance of a full size tracking detector at the LHC. The performance of the first generation prototype was reported in [5].

\section{The Transition Radiation Tracker (TRT) Prototype}

\subsection{Global detector principle}

The active detector elements of the TRT prototype are thin cylindrical proportional chambers, referred to as straw chambers or straws. These detectors, proposed in [6], are robust wire chambers because of the autonomy of each anode wire.

The straw size had to be chosen taking into consideration the expected LHC particle density, bunch crossing frequency and maximum drift time in the straws. There also exists a practical limit of roughly $3 \mathrm{~mm}$ for the smallness of the straw diameter. A $4 \mathrm{~mm}$ straw diameter, reasonable for a detector that will be produced in large quantities, was therefore chosen. Interleaving the straw chambers with radiators to produce transition radiation X-rays, and using Xenon in the chamber gas mixture to detect these, gives a combined transition radiation and tracking detector, a TRT, i.e. a tracker with enhanced electron identification capability $[7,8,9]$.

A tracking detector based on this principle is foreseen at an LHC experiment with a solenoidal magnetic field [10]. The radial coverage is expected to be about $0.5-1 \mathrm{~m}$. The combined constraint of measuring the azimuthal angle (solenoidal field) and that the particles should traverse the straws with as large an incident angle as possible, defines that the straws should be axial in the central region and radial in the forward region.

The prototype described here was tested at the $\mathrm{H} 6$ beam in the SPS at CERN from July to September 1991 and June to July 1992. A schematic layout of this prototype is shown in fig. 1. It consisted of 4 blocks of straws embedded in a polypropylene foam radiator of density $\sim 0.06 \mathrm{~g} / \mathrm{cm}^{3}[12]$. Each block contained 24 rows of straws with $8 \mathrm{~mm}$ distance between rows and straws within a row. The rows were randomly displaced with respect to one another to achieve uniform sensitivity of the detector, independently of the impact point of the beam. The total thickness of the detector amounted to $\sim 10 \%$ of a radiation length.

Block 1

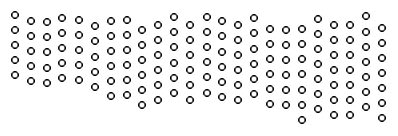

Block 2

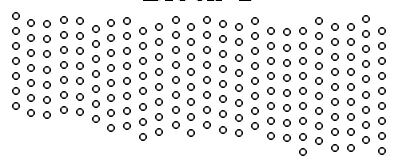

Block 3

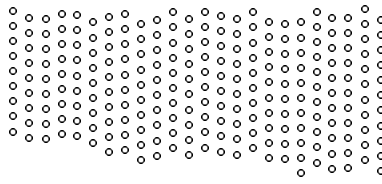

Block 4

Figure 1: Schematic layout of the RD6 Transition Radiation Tracker (TRT) prototype 
The straws were $40 \mathrm{~cm}$ long and $4 \mathrm{~mm}$ in diameter. A detailed description of their characteristics can be found in $[13,14]$. An additional block with $100 \mathrm{~cm}$ long straws was also tested but not used for the data analysis. The total number of straws was 864 for the 4 short blocks and 280 for the long block. The straws were operated with a $70 \% \mathrm{Xe} / 20 \% \mathrm{CF}_{4} / 10 \% \mathrm{CO}_{2}$ gas mixture at a gas gain of $2.5 \times 10^{4}$. This mixture combines the advantages of efficient TR-absorption, short drift time and stability with respect to discharges. The total drift electron collection time was approximately 34 ns without magnetic field and $38 \mathrm{~ns}$ in the presence of a $2 \mathrm{~T}$ magnetic field $[13,14]$.

\subsection{Experimental setup}

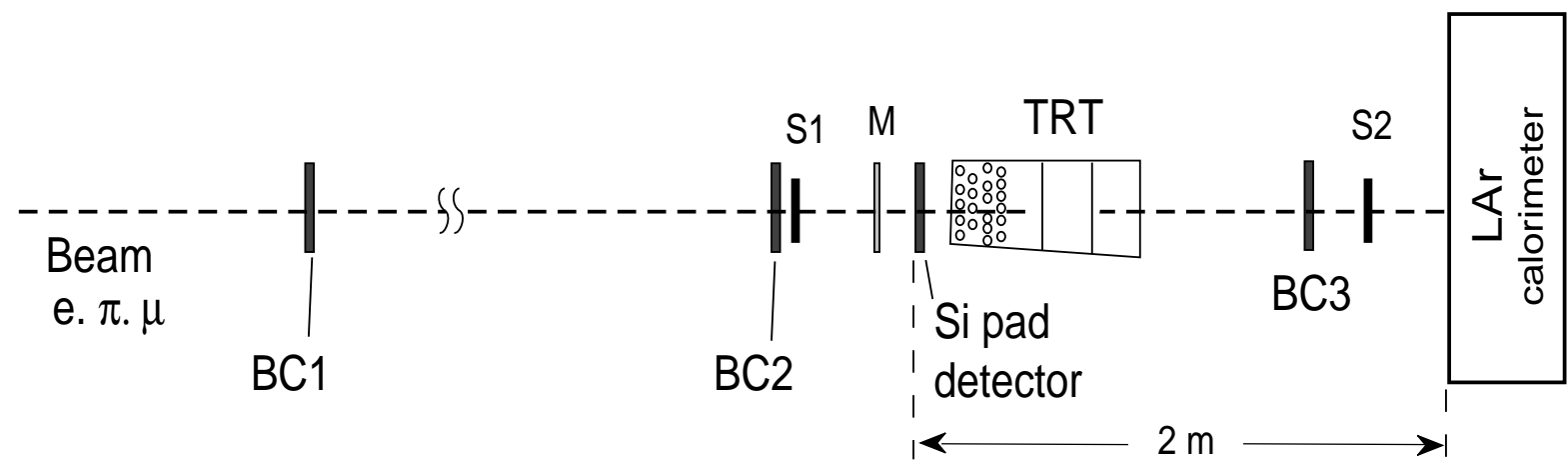

Figure 2: Experimental setup in 1991

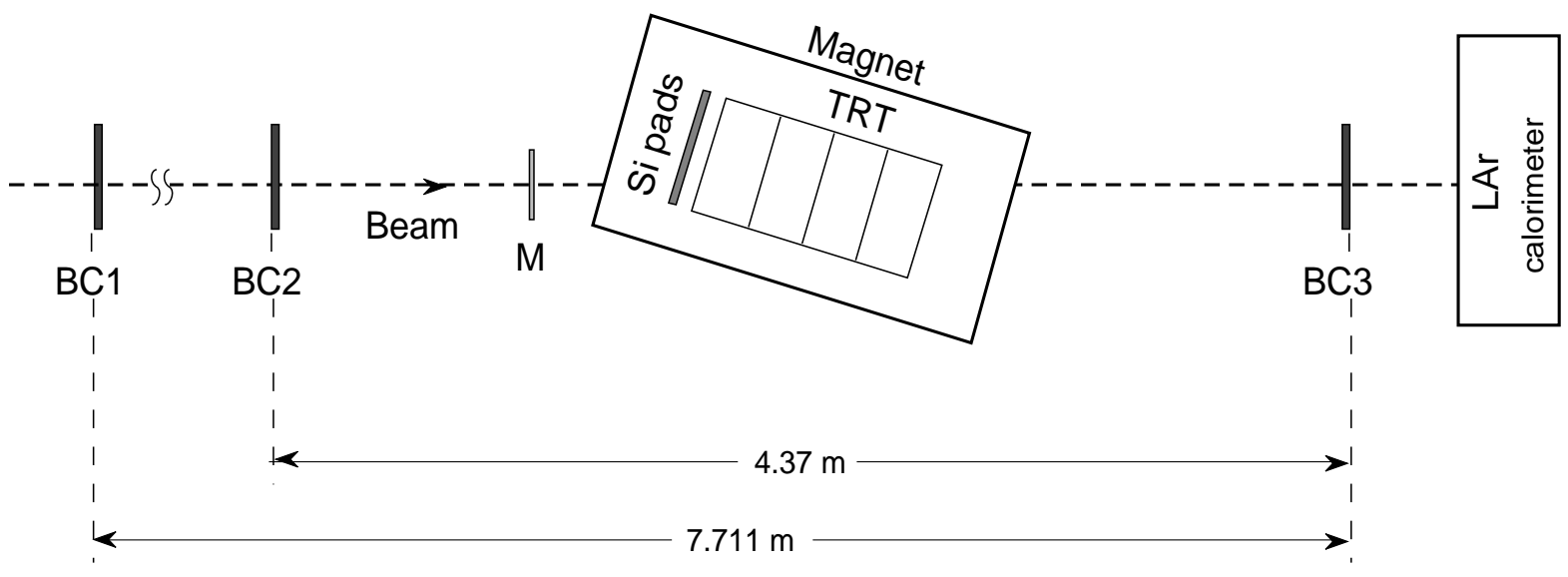

Figure 3: Experimental setup in 1992

Figure 2 shows a schematic layout of the overall setup for the 1991 run and fig. 3 the layout for the 1992 run, where the performance of the prototype was tested in the presence of a magnetic field. As can been seen from figs. 2 and 3 , the setup included scintillation counters for triggering, beam chambers for beam particle track reconstruction with space resolution $\sigma=330 \mu \mathrm{m}$, a silicon pad detector with 189 pads of $1.2 \times 5 \mathrm{~mm}^{2}$ size for tagging close-by charged particles and a prototype liquid argon calorimeter, which was used to reconstruct electromagnetic showers with good energy and position resolution. A detailed description of this prototype liquid argon calorimeter can be found in [15] and [16]. The calorimeter area used for the RD6 run consisted of $6 \times 9$ cells of $2 \times 2 \mathrm{~cm}$, with two compartments in $z$ (beam direction), equipped with cold GaAs preamplifiers. Each compartment amounted to $12.5 \mathrm{X}_{0}$. In 1991 the setup also included a hadronic calorimeter which consisted of two sections of the former HELIOS uranium-liquid Argon (ULAC) calorimeter [17]. In 1992 the setup also included a liquid argon preshower prototype [18].

The analogue electronics chain for the straws followed the design described in [19]. It consisted of preamplifiers mounted near the ends of the straws and connected to fast shaping amplifiers. The charge sensitive preamplifier had a common base input followed by two emitter followers. The rise time (10\% to $90 \%$ ) was $2 \mathrm{~ns}$ and the decay time was $40 \mathrm{~ns}$. There were three input channels in each hybrid. The shaper 
output had a $20 \mathrm{~ns}$ width at the base, and a $7 \mathrm{~ns}$ rise time for signals from $5.9 \mathrm{keV}$ photons. The output of the shaper was split: one output went to charge integrating analog-to-digital converters (Lecroy 2282 Camac modules with 48 channels/module) recording the energy deposition in the straw, and the other to fast discriminators with a software selectable threshold in the region from 0.15 to $10 \mathrm{keV}$ (CAMAC module with 24 channels/module), which were used to count TR-clusters, i.e. signals with energy above a preselected threshold. The electronics noise level was about $200 \mathrm{eV}(4 \sigma)$ at the input of the preamplifier. No drift-time information was recorded for these measurements.
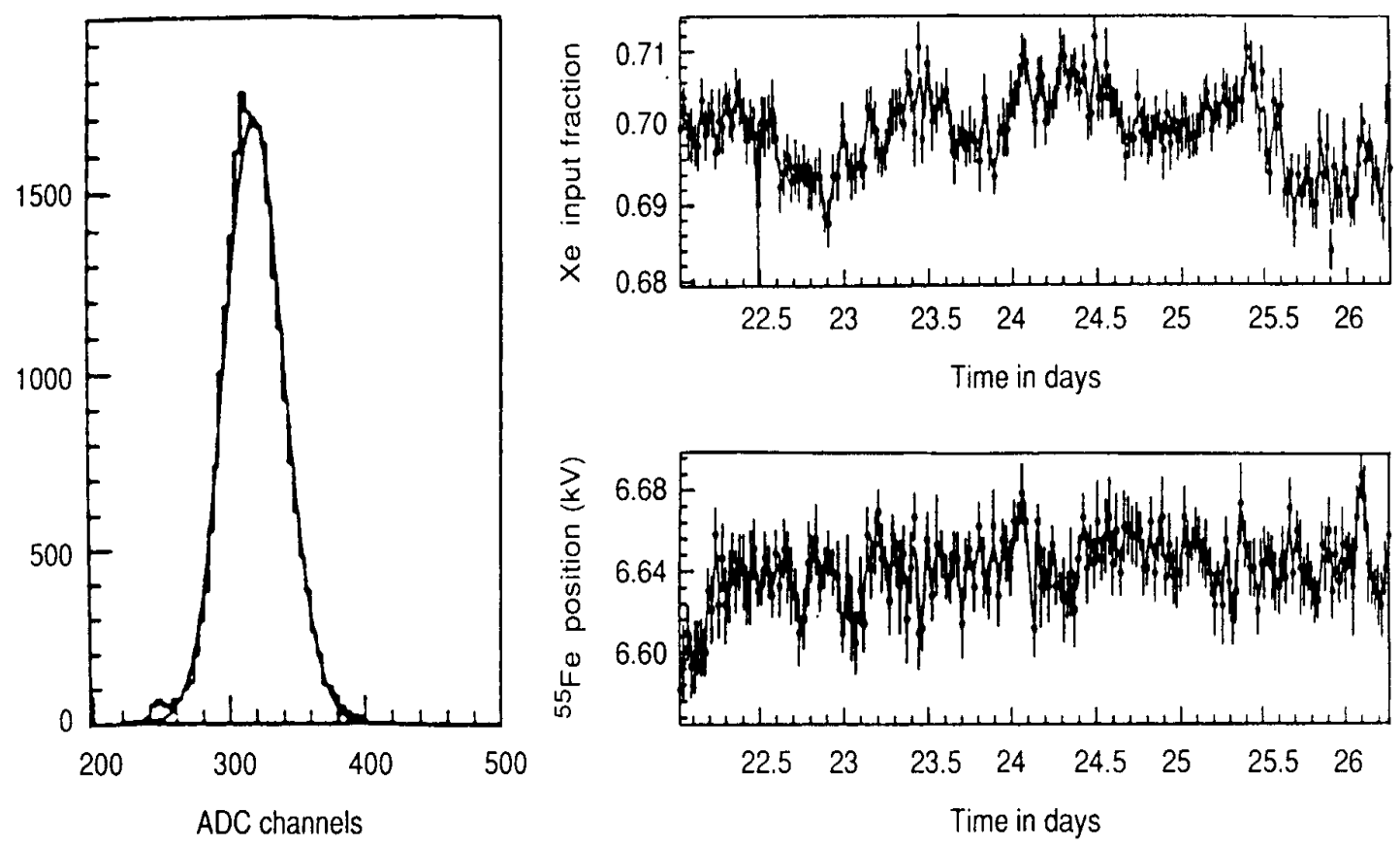

Figure 4: a) ${ }^{\mathbf{5} 5} \mathrm{Fe}$ pulseheight as measured by a straw and b) Variation with time of Xenon concentration and gas gain in the 1991 prototype

The Xenon concentration and the gas gain were carefully monitored throughout the run. The gas gain was stabilised by varying the operating voltage as a function of the ambient temperature and atmospheric pressure, using as a feedback signal the pulse height measured by a set of monitoring straws exposed to an ${ }^{55} \mathrm{Fe}$ source. Figure 4 shows the observed variations in gas gain over a period of 4 days, demonstrating that the dispersion after feedback correction was less than $\pm 1 \%$. Similarly, the Xenon concentration was monitored using ratios of counting rates from two straws exposed to an ${ }^{55} F e$ source. These straws were arranged in such a way that the first one shadowed the other from the source and thus gave information about the TR-photon absorption probability in the gas. Figure 4 b) shows that the concentration variations were below $\pm 1 \%$.

The ADC pedestals were obtained with a random trigger taken outside the burst. The absolute energy calibration of all the channels was done with an ${ }^{55} \mathrm{Fe}$ source, at the beginning and the middle of the data taking period. An example of the pulse height distribution for ${ }^{\mathbf{5} 5} \mathrm{Fe} 5.9 \mathrm{keV} \mathrm{X}$-rays as measured by a straw is shown in fig. 4 .

\section{Charged Hadron Rejection in the Absence of Magnetic Field}

The rejection against charged hadrons was measured using a $20 \mathrm{GeV}$ hadron beam, which consisted mainly of $\pi^{+}$, and a separate $30 \mathrm{GeV}$ electron beam to monitor the efficiency. The possibility of using the TRT for muon identification was investigated using muon beams with energy from 90 to $200 \mathrm{GeV}$. The dependence of the hadron rejection on various detector parameters, such as the detector thickness, the angle between the straws and the beam, the gas composition, the energy threshold for TR-clusters, the straw gain dispersion and the charge collection time, was also studied. 


\subsection{Event selection}

Clean beam triggers were defined as having one and only one particle inside the TRT aperture. Candidates were selected by applying a cut on the amplitude of the multiplicity counter and requiring a single hit in the Silicon pad detector, with charge compatible with that from one minimum ionising particle. Straight tracks pointing inside the TRT aperture were reconstructed using the $x$ and $y$ coordinates of the hits in the beam chambers $(\mathrm{BC} 1, \mathrm{BC} 2$ and $\mathrm{BC} 3)$ and the Silicon pads. After this initial selection, specific calorimeter cuts were applied, depending on the nature of the particle to be selected.

Electrons of $30 \mathrm{GeV}$ were required to have one and only one reconstructed electromagnetic shower with energy greater than $28 \mathrm{GeV}$. The shower position was required to match the one obtained by extrapolating the reconstructed beam particle track within $\pm 3 \mathrm{~mm}$ in $x$ and $\pm 4 \mathrm{~mm}$ in $y$. A longitudinal shower development cut, requiring that $85 \%$ of the shower energy be deposited in the first compartment of the electromagnetic calorimeter was also applied. As a transverse shower development cut, the ratio of the energy deposited in a matrix of $3 \times 3$ calorimeter cells over that deposited in a matrix of $5 \times 5$ cells was required to be greater than $85 \%$.

Pions of $20 \mathrm{GeV}$ were required to have deposited in the hadronic calorimeter energy significantly higher than that expected for a minimum ionizing particle. The total energy in the calorimeter, i.e. the sum of the total energy deposited in the electromagnetic part and in the hadronic compartment, was required to be greater than $2 \mathrm{GeV}$. Events with one reconstructed electromagnetic shower, with more than $80 \%$ of the energy deposited in the first calorimeter compartment in the $z$ direction, and the ratio of energy summed in a matrix of $3 \times 3$ cells over that in a matrix of $5 \times 5$ cells greater than $80 \%$ were classified as ambiguous and rejected. The final electron contamination in the pion sample was measured to be less than $10^{-4}$.

About $80 \%$ of electrons and pions satisfied the above criteria and were retained.

Muons were required to have less than $0.8 \mathrm{GeV}$ total energy deposited in the hadronic calorimeter and less than $1 \mathrm{GeV}$ in the electromagnetic calorimeter.

\subsection{Track finding in the TRT prototype}

As mentioned above, only the four blocks of $40 \mathrm{~cm}$ long straws were used in this analysis. The total number of straws amounted thus to 864 , out of which $7 \%$ were not operational. Before proceeding to track reconstruction based on the signals registered by the straws, noisy channels, i.e. channels with hit frequency higher than $40 \%$, were identified and excluded.

The overall alignment of the straws was done with experimental data, using the information from the beam track as reconstructed by the beam chamber and silicon pad detector hits. The straws were aligned to an accuracy of $100 \mu \mathrm{m}$ after this procedure was applied.

Straws with recorded energy above $0.2 \mathrm{keV}$ were then searched for in a road progressively narrowed down to $\pm 2.2 \mathrm{~mm}$. A straight line was then fitted to a minimum of 20 such hits and the tracking accuracy was finally optimized by maximizing the number of hit straws (i.e. straws with energy deposition above $0.2 \mathrm{keV}$ ) minus the number of 'empty straws' (i.e. straws with no hit registered) along the track. A detailed description of the alignment and tracking procedures can be found in [20]. Event displays of typical reconstructed electron and pion tracks are shown in fig. 5 .

By comparing the reconstructed TRT track with the one defined by the beam chambers and the silicon pad detector, the angular accuracy of the prototype was measured to be $0.45 \mathrm{mrad}$. By comparing the track defined by the 'odd' straws with the one defined by the 'even' straws on the original track, (the beam chamber resolution of $\sim 330 \mu \mathrm{m}$ being inadequate for this purpose), the overall position accuracy was measured to be $180 \mu \mathrm{m}$ at the centre of the detector. The deviation of hits from the reconstructed track is plotted in fig. 6 . The tails beyond $2 \mathrm{~mm}$ are due to extra hits from $\delta$-electrons and noise. An average of 41 hits were reconstructed per particle track, as shown in fig. 7 .

\subsection{Electron identification and comparison with Monte-Carlo simulation}

Figures 8 and 9 show the energy distributions of hits in straws along the reconstructed TRT track, for electrons and pions respectively. The data are compared to a Monte-Carlo simulation of the test beam setup. This simulation was performed using the GEANT package, taking into account all relevant processes (hadron interactions, bremsstrahlung, photon conversions, multiple scattering, $\delta$-ray production), as well as some processes not adequately included in GEANT, but essential for our simulations, such as transition and synchrotron radiation and ionisation in thin gas layers [21].

Electron identification makes use of the difference in the energy deposited in the straws by hadrons and electrons, and in particular of the higher tail above $4 \mathrm{keV}$. For electrons, this tail is due to transition radiation hits, while for pions, it is largely due to $\delta$-rays. Therefore, a very good accuracy of the simulations is crucial in order to correctly predict the hadron rejection. The agreement between the measured and 


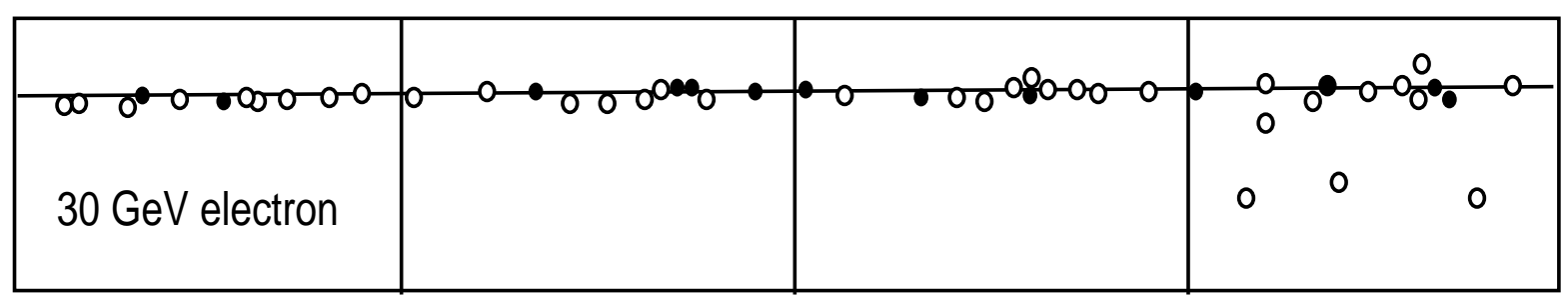

\begin{tabular}{|c|c|c|c|}
\hline $\begin{array}{l}0,0 \text { - } 0-0-80-\infty \\
20 \mathrm{GeV} \text { pion }\end{array}$ & $-\infty{ }^{\infty}$ & $-00-0$ & $+\infty 00-\infty 0-0<0-0-0-0$ \\
\hline
\end{tabular}

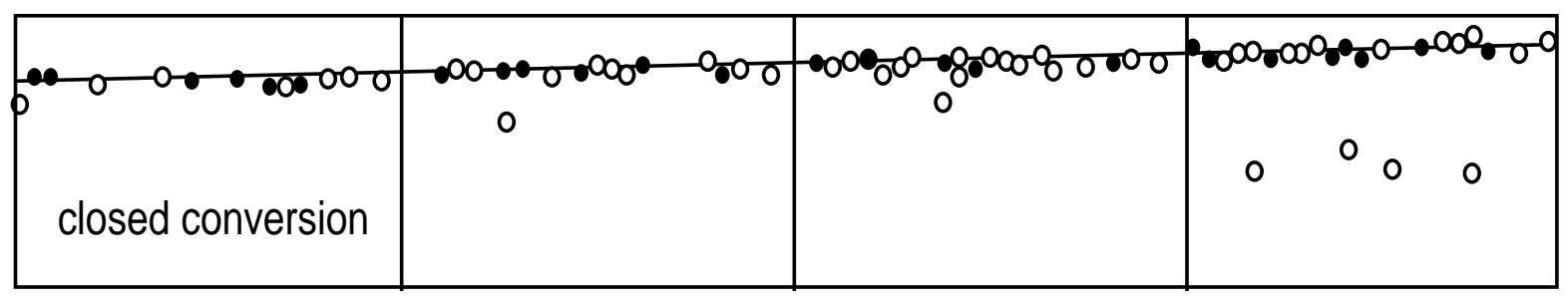

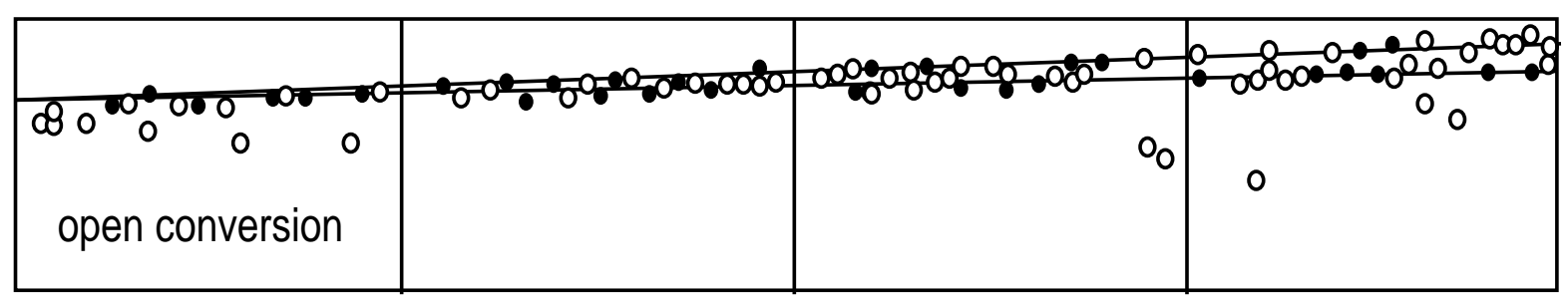

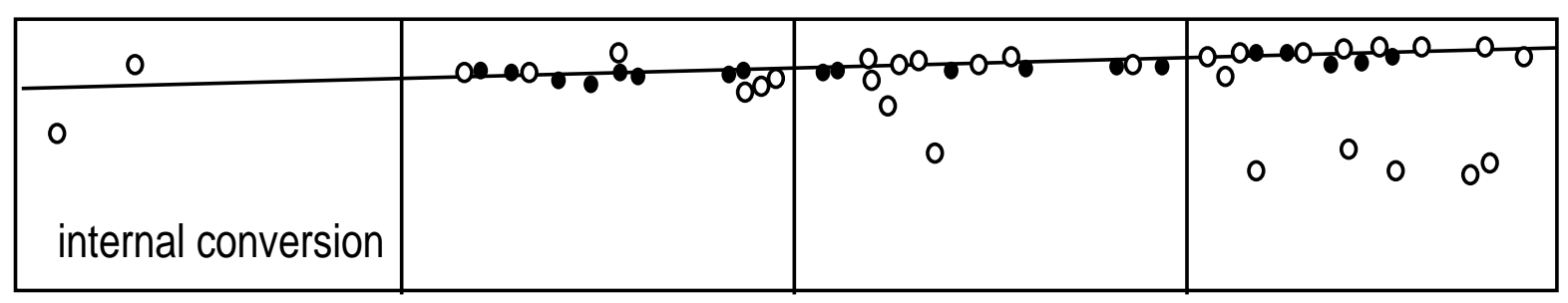

Figure 5: Event display in the TRT prototype: a) $30 \mathrm{GeV}$ electron b) $20 \mathrm{GeV}$ pion c) 'closed' conversion d) 'open' conversion e) conversion in the TRT material. The open (full) circles represent straws with energy deposition between $0.2 \mathrm{keV}$ and $5 \mathrm{keV}$ (larger than $5 \mathrm{keV}$ ). 


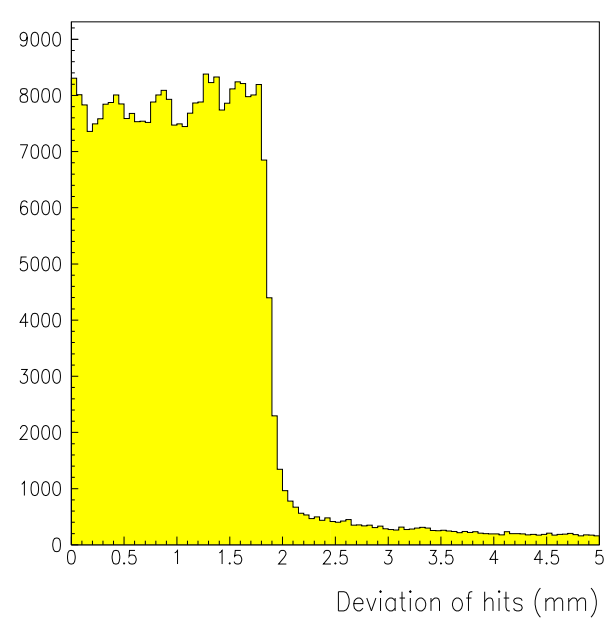

Figure 6: Deviation of hits from reconstructed TRT track

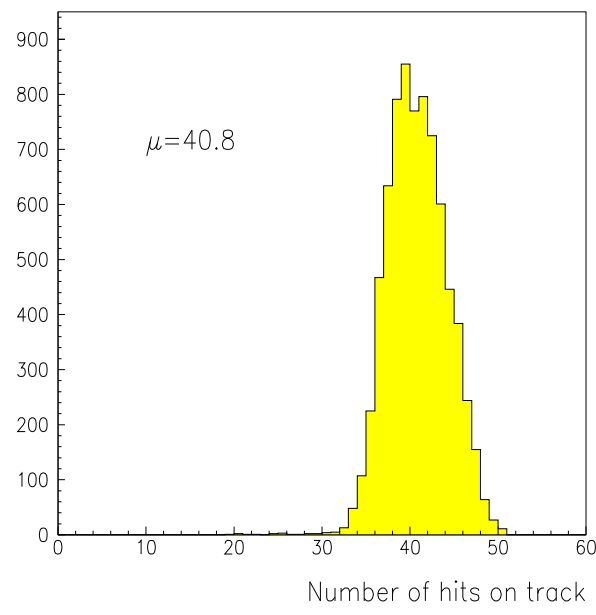

Figure 7: Number of hits along reconstructed TRT track

the simulated distributions is good and the small difference in the 4-6 keV range for electrons is due to different radiator structures: the RD6 measurements were taken with an irregular foam radiator while the simulations were done for a stack of regular foils.

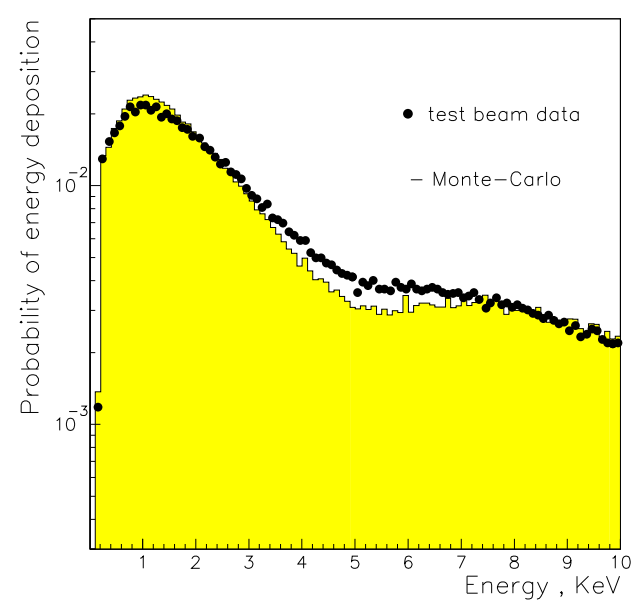

Figure 8: Energy distribution of hits on TRT track for $30 \mathrm{GeV}$ electrons

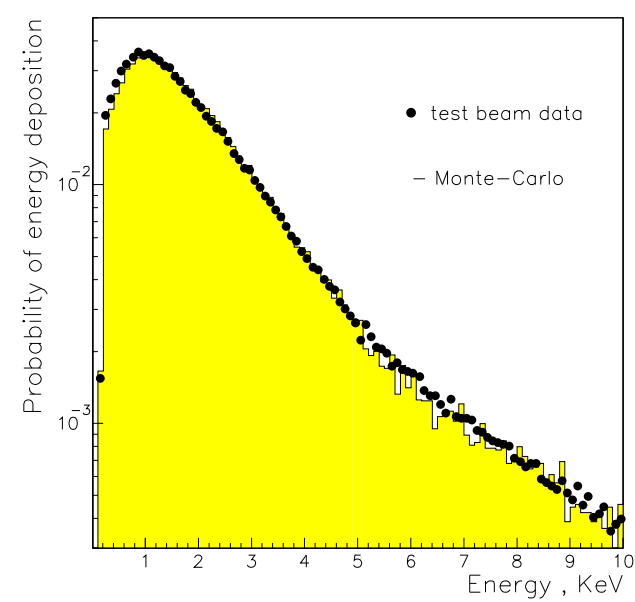

Figure 9: Energy distribution of hits on TRT track for $20 \mathrm{GeV}$ pions

The hadron rejection is calculated by counting the number of transition radiation (TR) clusters (i.e. hits with energy $E_{h i t}>E_{t h r}$ ). Events with few TR clusters were rejected, thus leading to a pion contamination which was a function of the chosen electron efficiency (usually 90\%). For the studies described here, the energy threshold, above which an energy deposition was accepted as a transition radiation cluster, was chosen to be $5.5 \mathrm{keV}$. The corresponding distributions of the number of clusters obtained for electrons and pions are shown in fig. 10. From fig. 10, for $90 \%$ electron efficiency, the remaining pion contamination is $0.37 \% \pm 0.06 \%$, i.e. a rejection factor of $\sim 300$ was achieved against $20 \mathrm{GeV}$ pions.

The energy threshold used to define transition radiation clusters along the reconstructed track can be optimized in terms of the hadron rejection at the chosen electron efficiency of $90 \%$. The efficiency for $20 \mathrm{GeV}$ pions as a function of this threshold is shown in fig. 11. The optimum threshold is approximately $6.5 \mathrm{keV}$, but the pion efficiency varies slowly for thresholds between 5 and $8 \mathrm{keV}$. As a consequence, a $10-20 \%$ channel-to-channel dispersion of the overall straw gains would not significantly degrade the 


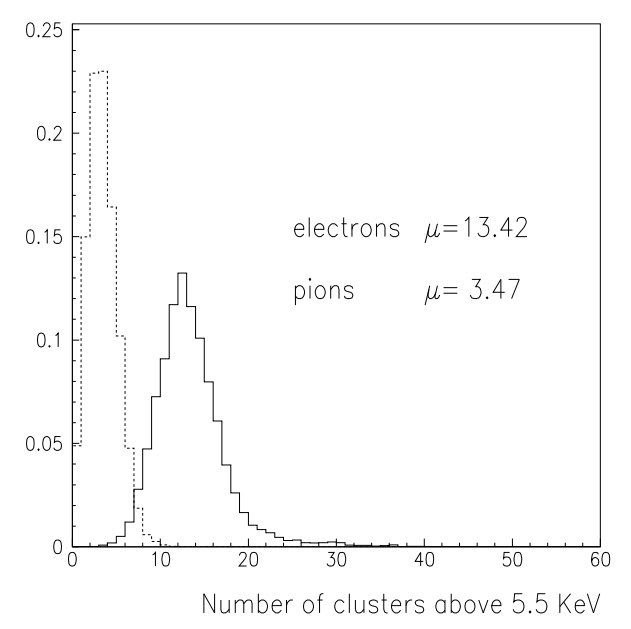

Figure 10: Number of clusters above $5.5 \mathrm{keV}$ for $30 \mathrm{GeV}$ electrons (full line) and $20 \mathrm{GeV}$ pions (dashed line)

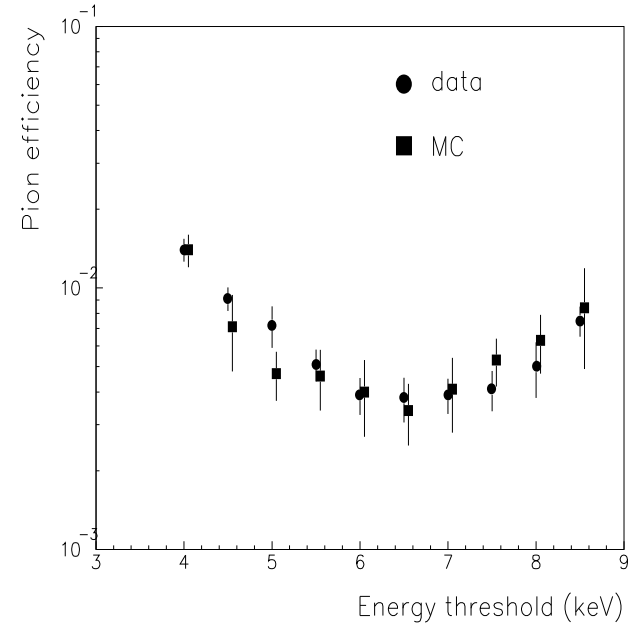

Figure 11: Pion efficiency versus energy threshold for TR-clusters at $B=0$

electron/pion separation. In addition, these measurements have shown that calculating the rejection by counting the number of clusters with energy above an optimized threshold, with the use of fast discriminators, yields the same results as using ADC measurements of the energy depositions in each straw.

The very clean separation between electrons and pions can also be seen from the two-dimensional distributions of the number of hits corresponding to one minimum ionising particle $\left(N_{12}\right)$, i.e. energy depositions between $0.2 \mathrm{keV}$ and $1.5 \mathrm{keV}$, versus the number of high energy clusters $\left(N_{3}\right)$, as shown in figs 12 and 13.

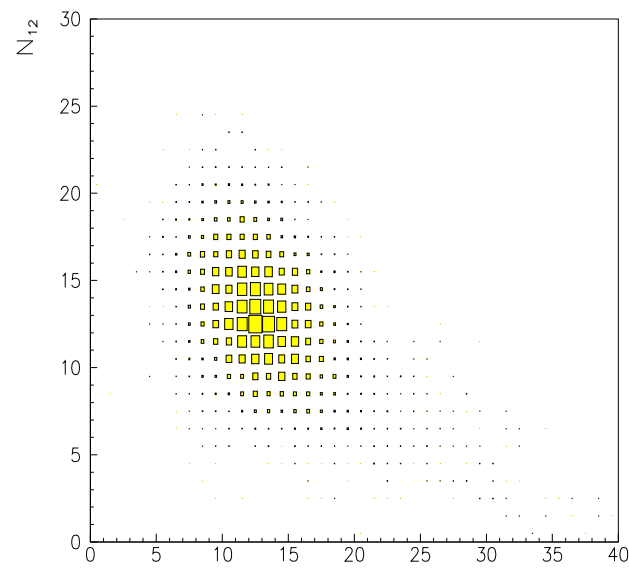

$N_{3}$

Figure 12: $N_{12}$ versus $N_{3}$ for $30 \mathrm{GeV}$ electrons

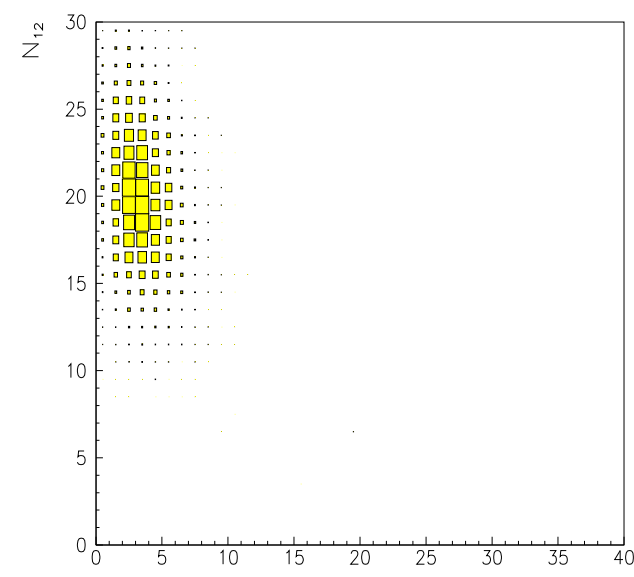

$N_{3}$

\subsection{Muon Identification}

Muon identification was studied in a similar way to that employed for electrons. Figure 14 shows the pion contamination $\left(E_{\pi}=20 \mathrm{GeV}\right)$ for $90 \%$ muon efficiency as a function of the muon energy. Good separation between muons and pions can thus be achieved for muon energies above $130 \mathrm{GeV}$. Using pion, muon and electron beams of different energies, the yield of TR-clusters has been accurately measured as a function of $\gamma=E / m c^{2}$ as shown in fig. 15 . 


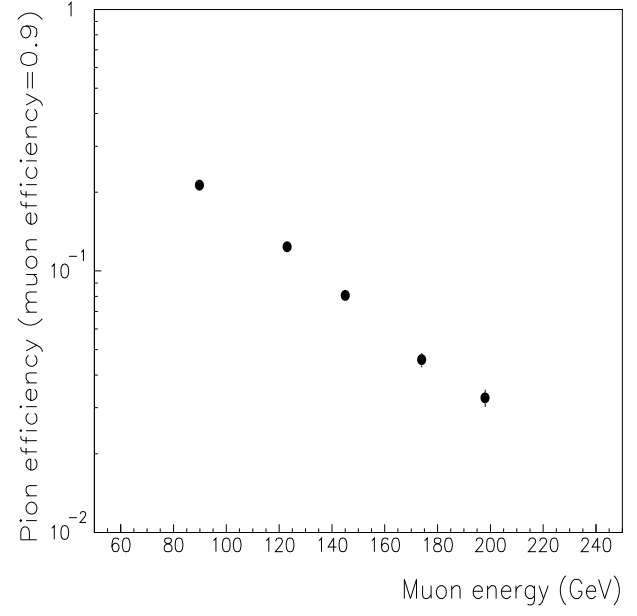

Figure 14: Pion efficiency versus muon energy

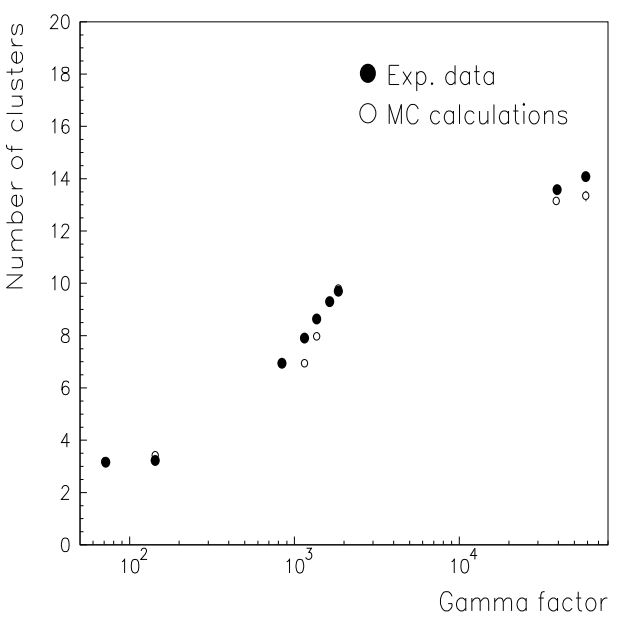

Figure 15: Number of TR clusters versus $\gamma=$ $E / m c^{2}$

\subsection{Dependence of Rejection on Detector Parameters}

- Detector thickness

The dependence of the rejection on the detector thickness was studied by counting the number of clusters over segments of the already reconstructed full-length track. Figure 16 shows the pion contamination at $90 \%$ electron efficiency as a function of the detector thickness, for three different tilt angles of the straws with respect to the direction of the incoming particles. A steep dependence of the rejection on the detector thickness is observed, since it improves by one order of magnitude as the detector thickness traversed by the particle increases from 40 to $80 \mathrm{~cm}$. The dependence on the angle is explained by the fact that the overall detector (depth, straw-radiator spacing etc) was optimized for $\mathrm{a} \sim 70^{\circ}$ angle.

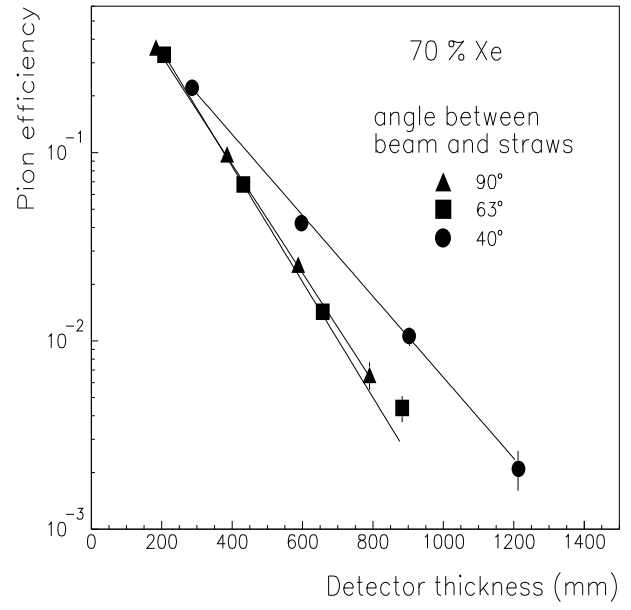

Figure 16: Pion efficiency versus detector thickness

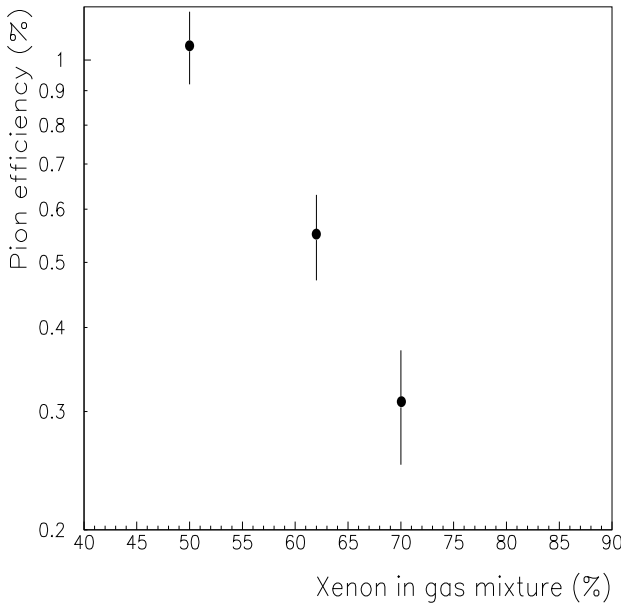

Figure 17: Pion efficiency versus Xe percentage in the gas

\section{- Gas composition}

As already mentioned, the straw gas consisted of Xenon, $\mathrm{CF}_{4}$ and $\mathrm{CO}_{2}$. As the percentage of Xenon increases, so does the probability of absorbing and therefore detecting a TR-photon. However, the maximum possible Xenon concentration is also determined by other considerations, such as the drift velocity and the operational stability of the straws with respect to discharges. For the three 


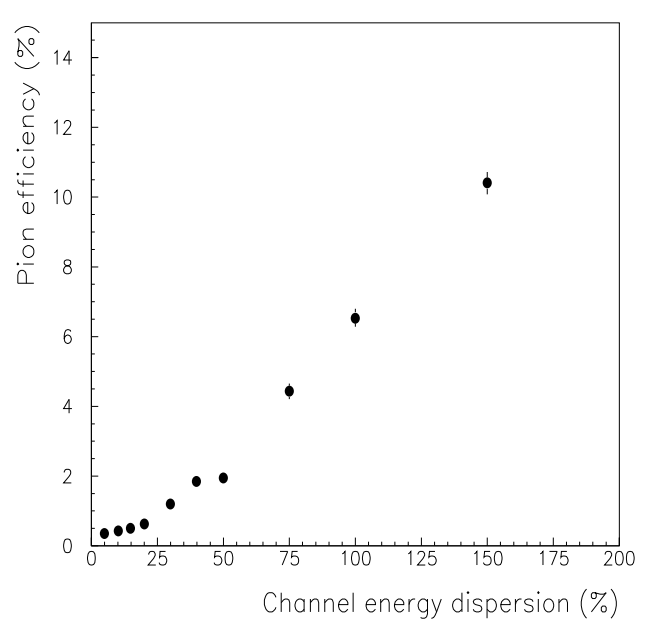

Figure 18: Pion efficiency versus channel gain dispersion

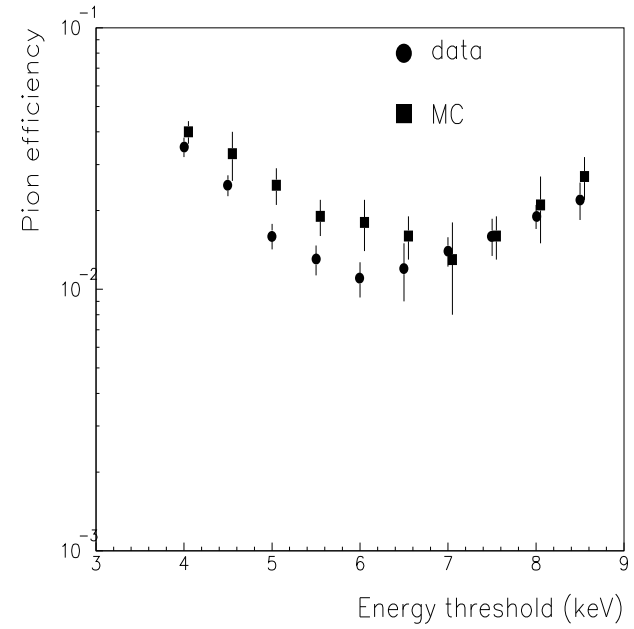

Figure 19: Pion efficiency versus energy threshold for TR-clusters in magnetic field

different gas compositions tested, $(50,62$ and $70 \%$ Xenon), the results are plotted in fig. 17. It can be seen that the rejection improves by a factor of $\sim 3$ as the Xenon concentration increases from 50 to $70 \%$.

- Channel gain dispersion

Any uncertainty in the straw energy scale may lead to a shift in the number of high energy clusters along the particle track and is therefore likely to affect the rejection. It is therefore important for the design of the electronics to determine what extent of channel gain dispersion is tolerable. The dispersion of the overall straw gains from channel to channel is the resultant of several ingredients (straw straightness, temperature, electronics gain ...) and the data were used to evaluate the sensitivity of the pion rejection to this dispersion. Each measured straw energy deposition was smeared with a given Gaussian r.m.s between 5 and $150 \%$. Figure 18, which shows the resulting variation of the pion efficiency as a function of this dispersion, demonstrates that the performance is not significantly affected for channel-to-channel dispersions less than $\sim 25 \%$.

\section{Charged hadron rejection in magnetic field}

The TRT prototype was also tested in the presence of a magnetic field during the 1992 run, in the setup shown in fig. 3. The setup comprised the RD6 TRT prototype placed inside the ALEPH TPC90 magnet (a solenoid with magnetic field $B=0.78 \mathrm{~T}$ along the magnet axis, corresponding to a field transverse to the incoming particle of $\mathrm{B}_{T}=0.257 \mathrm{~T}$ ).

\subsection{Event selection and track finding in the TRT}

The event selection criteria for this analysis were basically the same as for the single particle run without magnetic field (see Section 3.1), with the exception that only beam chambers BC1 and BC2 were used for the incident particle track reconstruction. The cut on the geometrical match of the reconstructed beam chamber track with the electromagnetic shower position in the calorimeter was applied taking into account the track shift in the magnetic field. The track finding algorithm for the TRT prototype in the magnetic field followed essentially the same procedure as described earlier. The only difference was that a straight line was fitted to the straws with energy above $0.2 \mathrm{keV}$ only at the first iterations of the algorithm and the final track was determined using a parabolic fit.

\subsection{Pion/electron separation and comparison with Monte-Carlo simulations}

The energy threshold used to define transition radiation clusters along the reconstructed track was again optimized in terms of the hadron rejection at the chosen $90 \%$ electron efficiency. The efficiency for $20 \mathrm{GeV}$ pions as a function of this threshold is shown in fig. 19, with a shape very similar to that in fig. 11 .

It should be noted however that the pion rejection appears to be three times better in the absence of magnetic field. Detailed Monte-Carlo simulations have shown that this apparently better rejection 


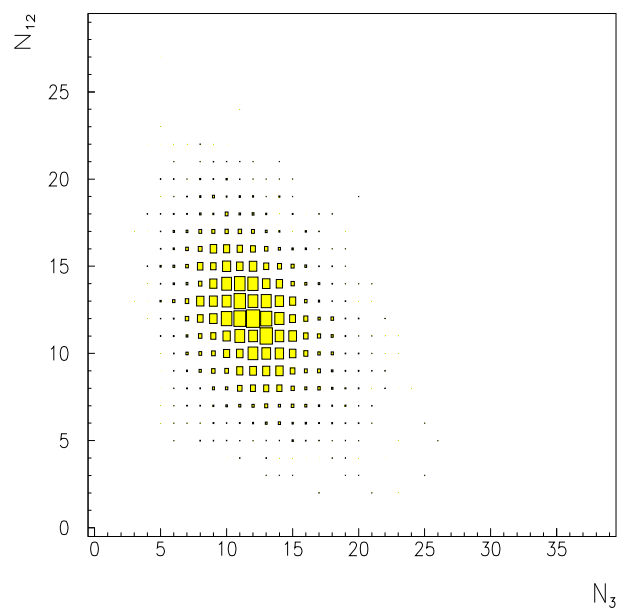

Figure 20: $N_{12}$ versus $N_{3}$ for 30 GeV electrons in magnetic field

is due to additional bremsstrahlung photons produced in the material upstream of the TRT prototype $\left(\sim 0.1 \mathrm{X}_{0}\right)$. These initiate early showers which accompany the particle trajectory and result in a $10 \%$ increase in the number of high energy clusters for electrons. We therefore conclude that the intrinsic rejection of $20 \mathrm{GeV}$ pions of the TRT prototype is a factor 100 (as measured in the presence of a magnetic field), and that it is sensitive to the amount of material upstream of the TRT (especially in the absence of magnetic field).
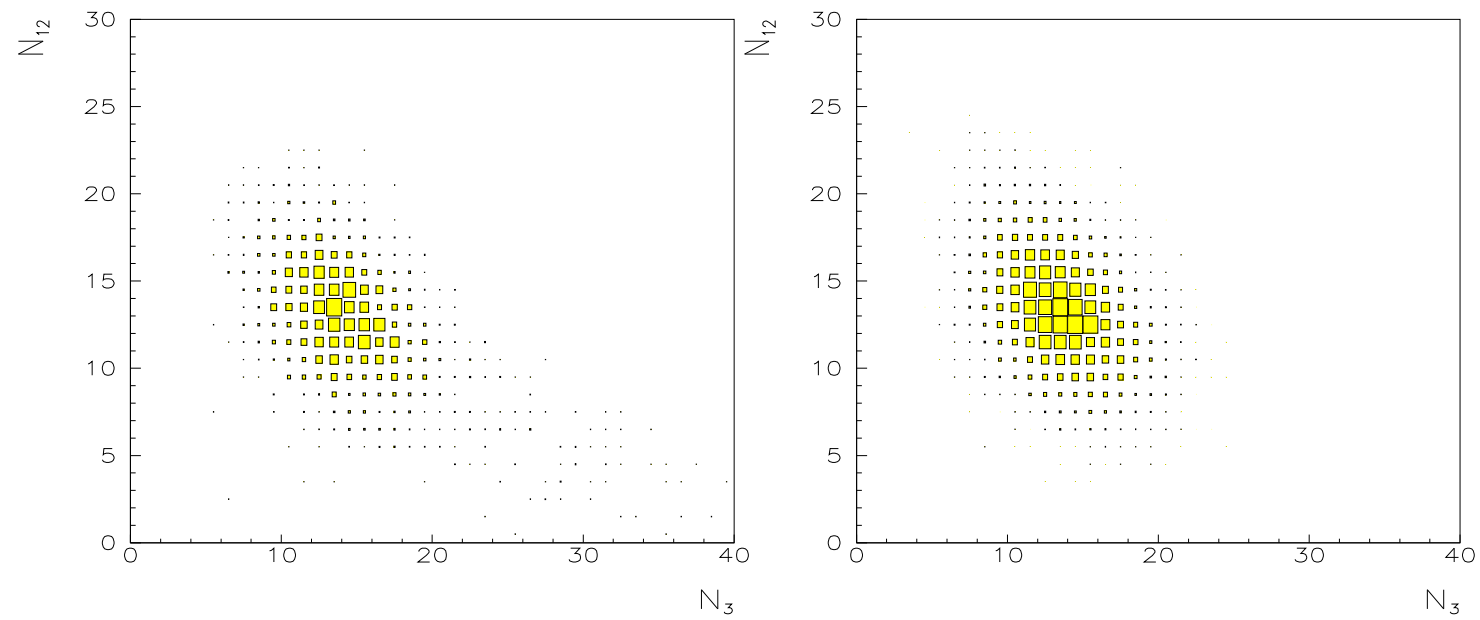

Figure 21: Electrons with and without bremsstrahlung (no magnetic field) in the upstream material (MC)

The tail at large $N_{3}$ and low $N_{12}$ consisting of such events can be clearly seen from the comparison of the two-dimensional distributions of the number of single particle hits versus the number of high energy clusters for $\mathrm{B}=\mathbf{0}$ (fig. 12) and $\mathrm{B}=0.78 \mathrm{~T}$ (fig. 20). The Monte-Carlo simulations, shown in fig. 21, display on the left-hand side, predictions including bremsstrahlung in the upstream material (which are in good agreement with the experimental data) and on the right-hand side, the predictions obtained with the bremsstrahlung in the upstream material switched off.

\subsection{Dependence of the rejection on the hit detection time}

At the LHC design luminosity, the straw occupancies are expected to be $\sim 15-20 \%$. The possibility of using a shorter hit detection time than the full $38 \mathrm{~ns}$ needed to collect all the drift electrons in the presence of the magnetic field, in order to minimize the TRT occupancy, has been studied using the 
experimental data. The idea is to register only the last $\sim 25 \mathrm{~ns}$ of the drift time, which corresponds to a radial position of energy deposition between 0.5 and $2 \mathrm{~mm}$ from the straw centre and therefore to $92 \%$ of the gas volume. For this purpose, the trailing edge of the gate is positioned at the end of the drift time. The deterioration of the pion rejection as a function of the total gate width (fig. 22) demonstrates that a gate of $25 \mathrm{~ns}$ could be used at the price of a factor 2 loss in the rejection power. More accurately defined strobes have been shown to significantly reduce the pile-up background at LHC design luminosity [22].

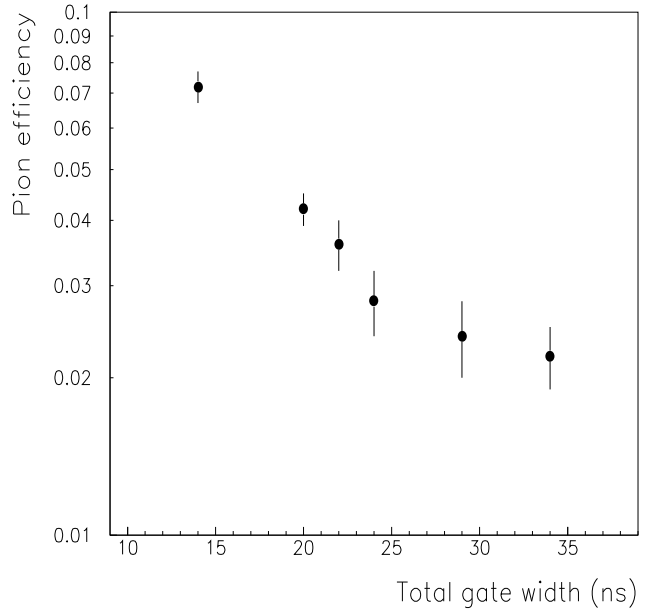

Figure 22: Pion efficiency versus total gate width

\section{Rejection of Photon Conversions in the Absence of Magnetic Field}

5.1 The Photon Beam Set-up

The rejection of photon conversions was studied with the same detector setup as the one described above. A beam of $150 \mathrm{GeV}$ electrons went through a $0.08 \mathrm{X}_{0} \mathrm{~Pb}$ converter as shown in fig. 23. The

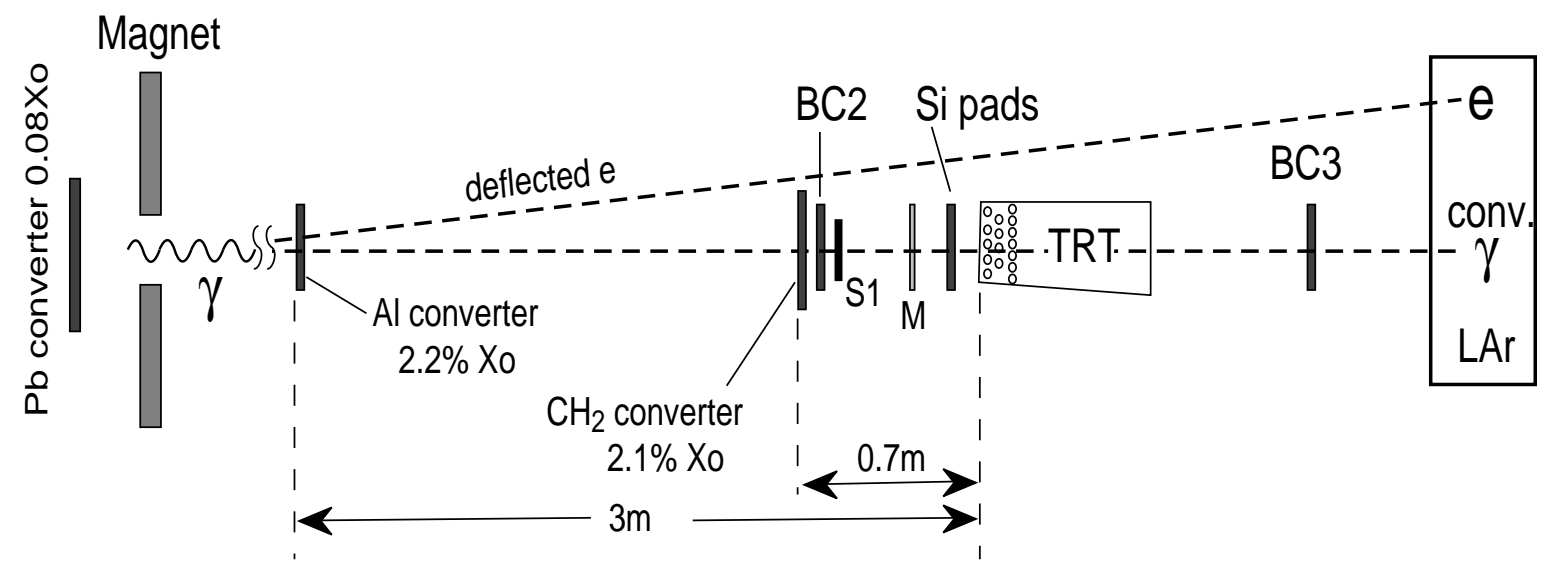

Figure 23: Photon Conversion setup in 1991

electron beam was subsequently deflected by a magnet towards the upper part of the LAr calorimeter without traversing the other detectors, while the triggered bremsstrahlung photons with energies between 20 and $140 \mathrm{GeV}$ and the characteristic $1 / E$ spectrum went through two converters upstream of the TRT. The first converter consisted of $2 \mathrm{~mm} \mathrm{Al}\left(2.2 \% \mathrm{X}_{0}\right)$, placed $300 \mathrm{~cm}$ upstream and the second consisted of $10 \mathrm{~mm} \mathrm{CH}_{4}\left(2.1 \% \mathrm{X}_{0}\right)$, placed $70 \mathrm{~cm}$ upstream.

\subsection{Event Selection and Classification}

Clean beam triggers were selected by requiring at least one reconstructed electromagnetic shower with a reconstructed position in the calorimeter compatible with that of a converted photon; as mentioned 
Table 1: Rejection of conversions with one and only one reconstructed TRT track, at $\epsilon_{e}=0.9$

\begin{tabular}{||c|c|c||}
\hline Type of conversion & $\epsilon_{c}(T R)$ & $\epsilon_{c}\left(T R, \chi^{2}\right)$ \\
\hline Closed & $\mathbf{0 . 0 3} \pm \mathbf{0 . 0 0 2}$ & $\mathbf{0 . 0 1 9} \pm \mathbf{0 . 0 0 1}$ \\
\hline Open & $\mathbf{0 . 1 0} \pm \mathbf{0 . 0 0 5}$ & $\mathbf{0 . 0 4 2} \pm \mathbf{0 . 0 0 3}$ \\
\hline
\end{tabular}

above, the incoming electron beam was deflected towards the upper part of the calorimeter, where a second shower was often measured. The events were also required to have at least one hit in the silicon pad detector. The converted photon track was measured by fitting all combinations of LAr showers, Silicon hits and beam chamber hits and selecting the one with the best $\chi^{2}$. These selection criteria were satisfied by $41 \%$ of the events.

The final conversion samples were then selected according to the number of hits in the silicon pads, their pulse height and their topology. The conversions from the nearest converter gave in most cases only one hit in the Silicon pads with a pulse height corresponding to two minimum ionising particles (mips), while those from the furthest one gave two single mip hits in the Silicon pads, thus mimicking assymetric pairs from Dalitz decays. A large fraction of these $(42 \%)$ had adjacent pads. As indicated by Monte-Carlo studies and expected in this setup, this sub-sample of events was the most background free and they were named 'open' conversions, while events with one hit with double pulse height were named 'closed' conversions.

\subsection{Track Finding in the TRT}

The track finding algorithm used in the case of single particles was adapted to search for multiple tracks. Only one track was reconstructed for $91 \%$ of the closed conversions. Open conversions were reconstructed as two tracks only in $11 \%$ of the cases, when the separation of the two electrons allowed the identification of two distinct tracks. It should be recalled here that the granularity of the Si pad detector was considerably finer than that of the TRT. Event displays for 'closed' and 'open' conversions are shown in fig. 5 .

\subsection{Rejection of Photon Conversions}

The rejection of photon conversions with one and only one reconstructed TRT track was calculated using the two-dimensional distributions of $N_{12}$ versus $N_{3}$ (figs. 24 and 25). The results are summarised in Table 1 for photons with energy larger than $10 \mathrm{GeV}$. The factor $\sim 3$ worse rejection for the so called 'open' conversions with one TRT track is due to the larger separation of the electron-positron pair inside the cluster counting road around the track. By counting clusters in a $\pm 5 \mathrm{~mm}$ road around the track instead of the $\pm 2 \mathrm{~mm}$ adopted for the analysis, this effect disappears and a rejection factor of $\sim 40$ can be achieved for these events as well as for those classified as closed conversions.

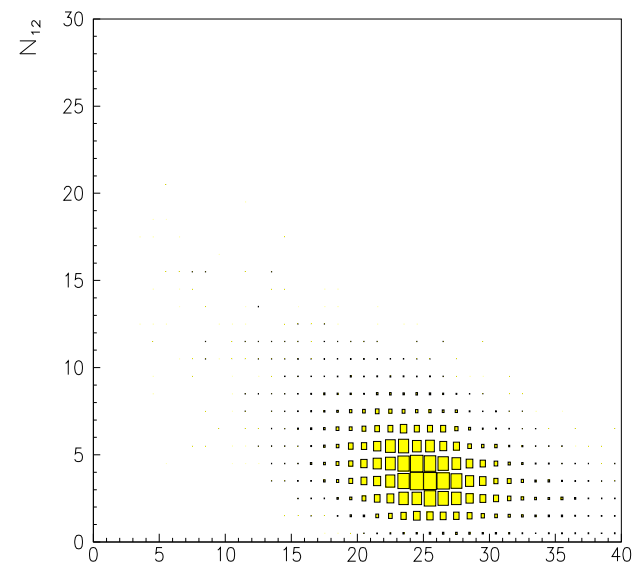

$\mathrm{N}_{3}$

Figure 24: $N_{12}$ versus $N_{3}$ for 'closed' conversions with one reconstructed TRT track

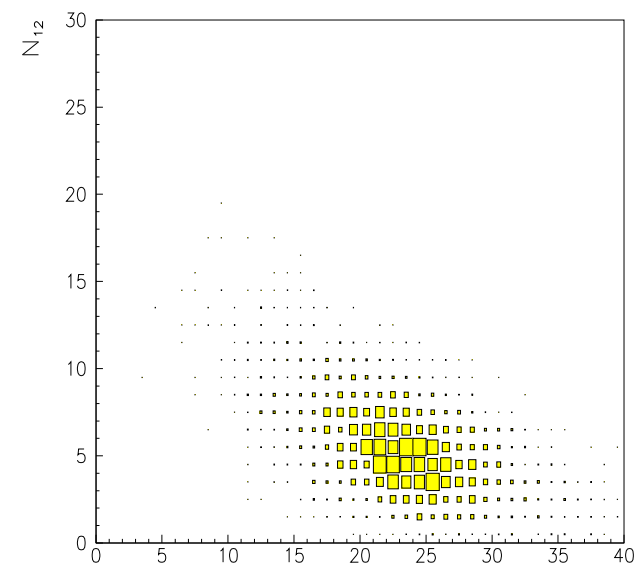

$N_{3}$

Figure 25: $N_{12}$ versus $N_{3}$ for 'open' conversions with one reconstructed TRT track 
The rejection of converted photons with one reconstructed TRT track can be improved if track quality criteria are combined with the TR-cluster information. As shown in table 1, this improves the rejection of closed (resp. open) conversions to values of $\sim 52$ (resp. $\sim 24$ ) for a $90 \%$ electron efficiency. Open conversions with two reconstructed TRT tracks can obviously be rejected if both tracks are identifiable as electrons and/or if they can be proven to originate from the same vertex.

\subsection{Dependence of Rejection on Photon Energy}

As mentioned above, the beam consisted of bremsstrahlung photons with energies ranging from 10 to $140 \mathrm{GeV}$. In order to study the conversion rejection as a function of the photon energy, sub-samples of events in six energy bins were used to calculate the rejection with the method described above. The results are plotted in fig. 26, for closed and open conversions with one reconstructed TRT track. As expected, the rejection improves as the photon energy increases, due to the smaller electron-positron angle of the conversion, which, in the absence of magnetic field, results in more hits inside the TRT tracking road.

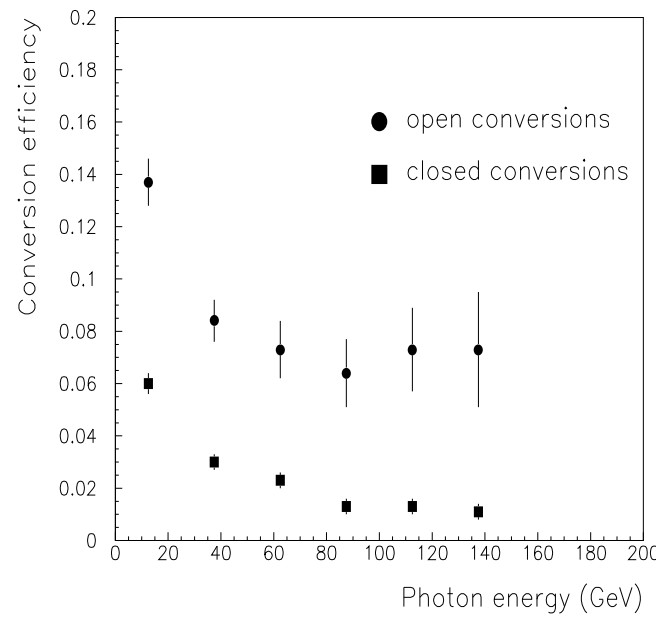

Figure 26: Conversion efficiency as a function of photon energy

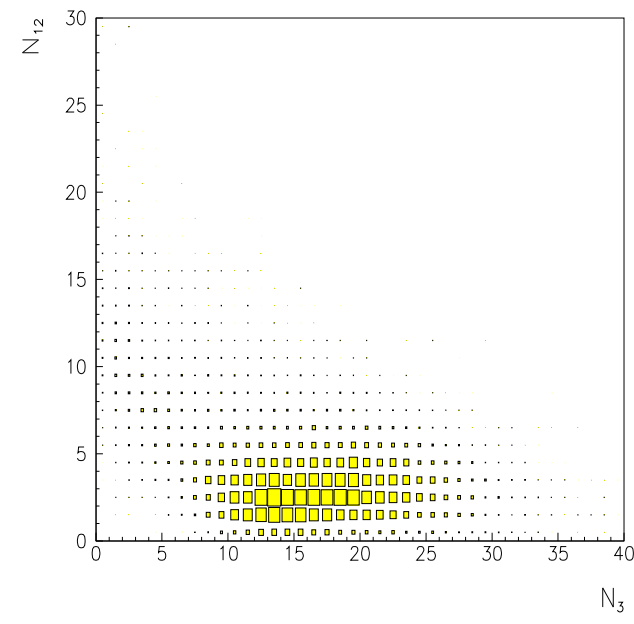

Figure 27: $N_{12}$ versus $N_{3}$ for photon conversions in the TRT material with one reconstructed TRT track

\section{Rejection of Photon Conversions Produced in the TRT Material}

In order to study the rejection of photon conversions produced inside the TRT, the original photon beam was directed into the detector, without traversing the two converters, while the parent electron beam was again deflected towards the upper part of the LAr calorimeter. Events were selected by requiring a conversion shower in the LAr calorimeter, as in the case of 'external' conversions, but with no hit in the silicon pads. About $46 \%$ of the events satisfied these criteria.

Tracking inside the TRT rejected about $58 \%$ of these conversion candidates, which failed to give a TRT track by not meeting the minimum criterion of 20 hits per reconstructed track. This was of course due to the fact that these conversions occurred deeper in the detector and had a considerably shorter path. As expected, in most of the cases $(\sim 90 \%)$, only one track was reconstructed, the electron-positron not having separated enough to produce distinct tracks. A typical conversion occurring inside the TRT is shown in fig. 5. Figure 27 shows $N_{12}$ versus $N_{3}$ for photon conversions in the detector material, with one reconstructed track. The rejection against those was calculated in the same way as for 'external' conversions. For $90 \%$ electron efficiency, the overall conversion efficiency was $\epsilon_{\gamma}=0.016 \pm 0.001$, which corresponds to a rejection factor of $\sim 62$.

\section{$7 \quad$ High multiplicity environment without magnetic field}

The TRT prototype was also tested in a target run in order to simulate the pile-up effects from minimum bias events at high LHC luminosities. The detector was positioned at an angle of about $40 \mathrm{mrad}$ in the vertical plane with respect to the incoming beam particles. The beam consisted of $205 \mathrm{GeV}$ pions and protons interacting in a thin beryllium target situated $70 \mathrm{~cm}$ in front of the TRT. The trigger was based on the coincidence of a large amplitude in the multiplicity scintillation counter and a shower with at 
least $10 \mathrm{GeV}$ energy observed in the LAr calorimeter behind the TRT. The display of one such triggered event is shown in fig. 28

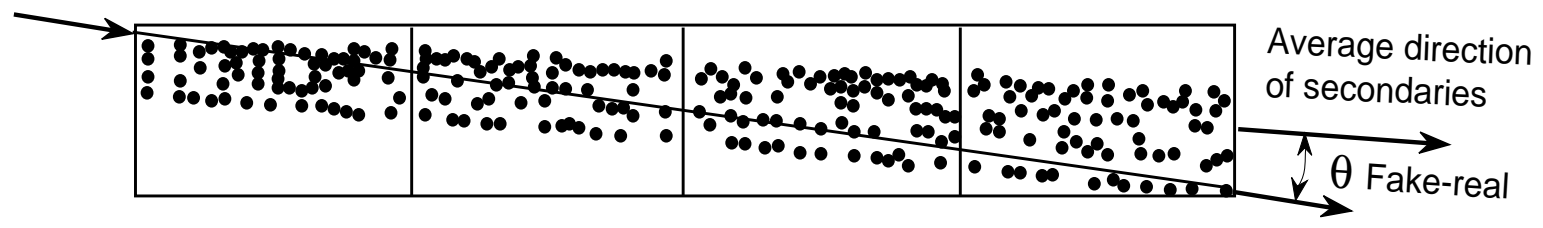

Figure 28: Display of a target run event, with a typical $1 \mathrm{~cm}$ wide road for fake track searches

\subsection{Electron identification}

A direct experimental measurement of the TRT prototype performance as a particle identification device in a high multiplicity environment was obtained by superimposing events from single particle runs on top of target run events. This method gave the possibility of monitoring the misidentification of the track parameters reconstructed with relatively high precision from the single particle run data (electrons or pions) as a function of the underlying occupancy originating from the hadronic interactions of incident pions and protons in the target.

A simple and robust reconstruction algorithm using the information around the superimposed track obtained from the single particle run was applied in order to minimize the sensitivity of the results to systematics in the track finding procedure. A road of $\pm 3 \mathrm{~mm}$ width with respect to the known single particle direction was scanned with angular steps of $2 \mathrm{mrad}$ and $1 \mathrm{~mm}$ in the $y$-coordinate. Among all the track candidates in the road, the one with the maximal difference between the number of hits $N_{\text {hit }}$ (i.e. straws with energy deposition above $0.2 \mathrm{keV}$ ) and the number of empty straws $N_{\text {empty }}$ was chosen. Once the track was established to first order, the $N_{h i t}-N_{e m p t y}$ quantity was maximized with a more precise iteration based on a Simplex algorithm. It looked for the minimum of a specially designed, weighted function which was the sum of individual straw weights. Each straw weight depended on the straw distance from the track candidate thus decreasing the impact of edge hits; the weight for an empty straw was added to the sum while the weight of a hit straw was subtracted:

$$
W_{i}=\frac{1}{1+\exp \left[\frac{d-r_{0}}{\sigma_{\text {edge }}}\right]}
$$

where $d=Y_{\text {track }}-Y_{i}, r_{0}$ the straw radius, and $\sigma_{\text {edge }}=50 \mu \mathrm{m}$ the $\sigma$ of the straw edge.

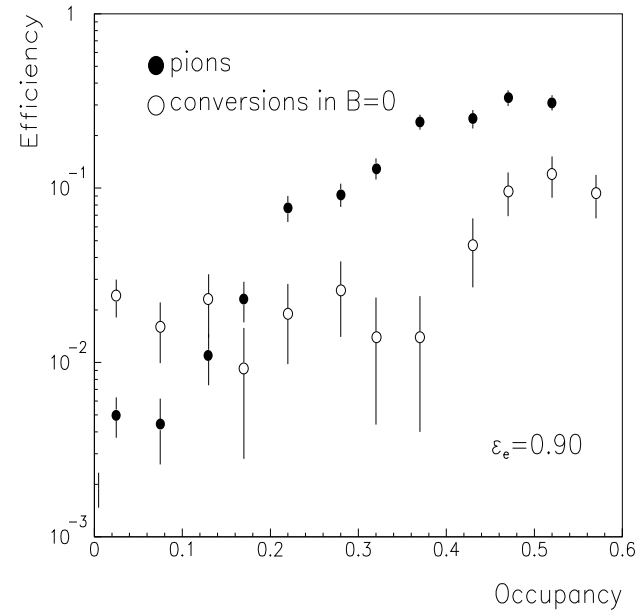

Figure 29: TRT pion and photon conversion efficiency versus occupancy

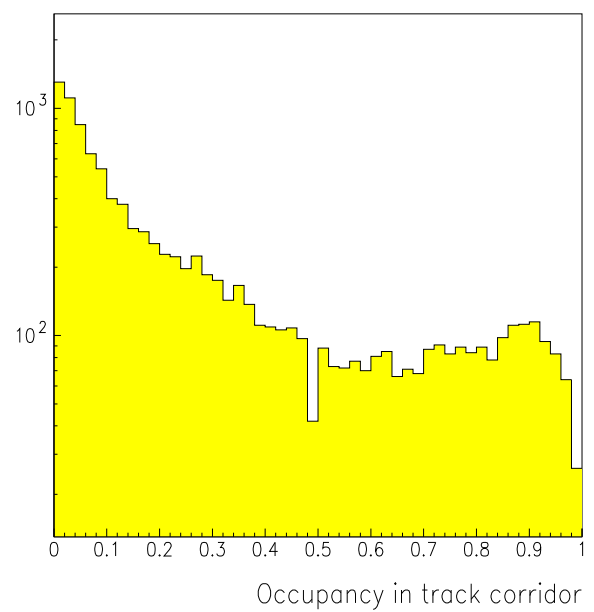

Figure 30: Straw occupancy distribution in target run data

The results of this study are shown in fig. 29 as the TRT pion and photon conversion rejection as a function of the straw occupancy. This occupancy is defined from the target run data as the ratio of the 
number of hits under the superimposed particle originating from the hadronic background over the total number of straws crossed by the reconstructed track. Figure 30 shows the measured distribution of this occupancy in the target run, which reaches values well above the highest straw occupancies foreseen in ATLAS at LHC. For the occupancy values expected at the LHC design luminosity (25\% average occupancy in the ATLAS barrel TRT and $15 \%$ in the endcaps) the photon conversion rejection is unaffected, whereas the pion rejection is worse by an order of magnitude than the one at very low occupancy.

\subsection{Study of fake track rates and comparison with Monte-Carlo simulations}
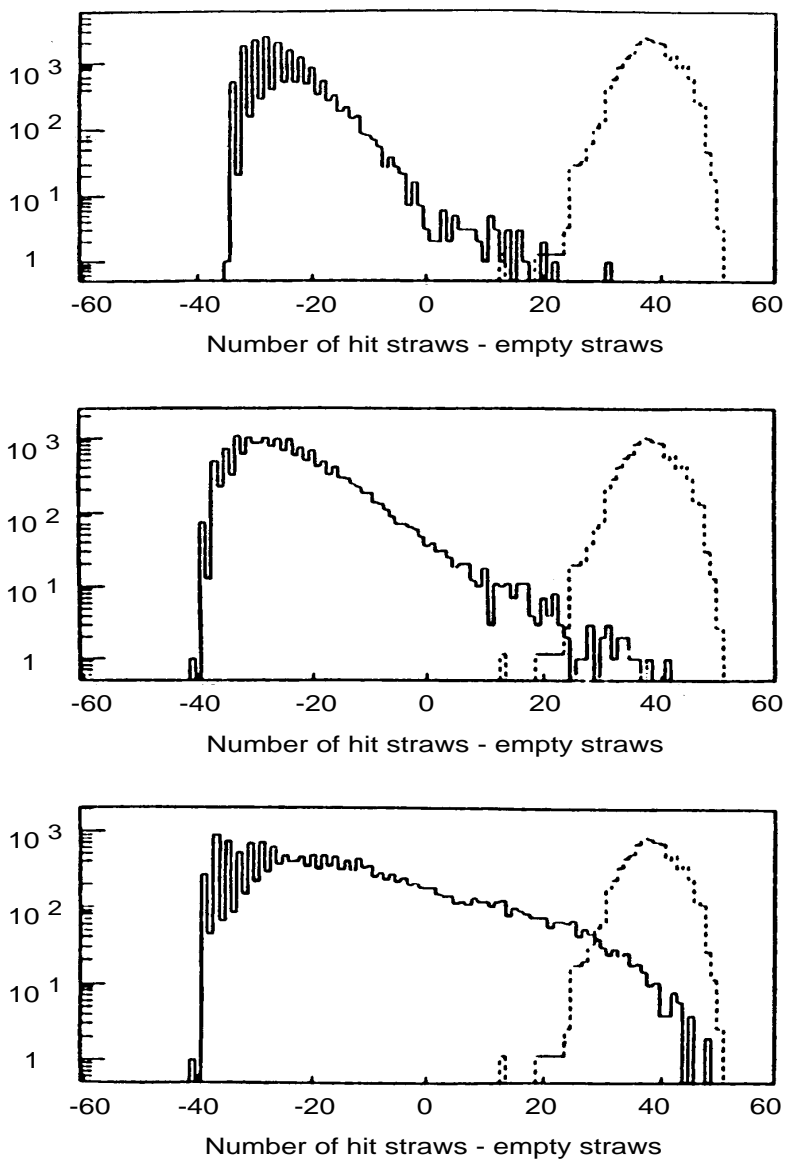

Figure 31: Distribution of the difference between the weighted numbers of hit straws and the weighted numbers of empty straws for real tracks with angle $\theta_{\text {real }}$ (dashed) and for fake tracks with angle $\theta_{\text {fake }}$ (solid), for increasingly smaller values of the difference $\theta_{\text {fake }}-\theta_{\text {real }}: 100 \mathrm{mrad}(\mathrm{a}), 50 \mathrm{mrad}$ (b) and $10 \operatorname{mrad}(c)$.

The TRT tracking performance in a high multiplicity environment was also studied in terms of the fake track rates for different detector occupancies.

The distributions of $N_{h i t}-N_{\text {empty }}$ and the weighted function for the track candidates were compared to the corresponding distributions obtained from the single particle runs. Figure 31 shows them for a $1 \mathrm{~cm}$ wide hit collection corridor and for three different values of $\theta_{\text {fake }}-\theta_{\text {real }}$, where $\theta_{f a k e}$ is the average fake track angle and $\theta_{\text {real }}$ the average angle of the real tracks originating from the target. These values correspond to different degrees of correlation between the hits from the real track and those in the fake track direction. For small values of $\theta_{f a k e}-\theta_{\text {real }}$ the correlation is the largest and corresponds to pattern recognition inside high- $\mathrm{p}_{T}$ jets or in a non-magnetic detector. For the ATLAS TRT at LHC, in a magnetic field of $2 \mathrm{~T}$, and for an average $\mathrm{p}_{T}$ of $0.6 \mathrm{GeV}$ for minimum bias tracks, the average value of $\theta_{f a k e}-\theta_{\text {real }}$ for isolated electron candidates is much larger, approximately $1000 \mathrm{mrad}$.

Figure 32 shows the dependence of the fake track probability on $\theta_{\text {fake }}-\theta_{\text {real }}$, for an average straw occupancy of $\sim 20 \%$, using the target run data and Monte-Carlo simulations to extrapolate to the expected LHC conditions. This figure demonstrates that the TRT pattern recognition capabilities are expected to be quite robust even at the highest LHC luminosity, provided that the number of crossed 


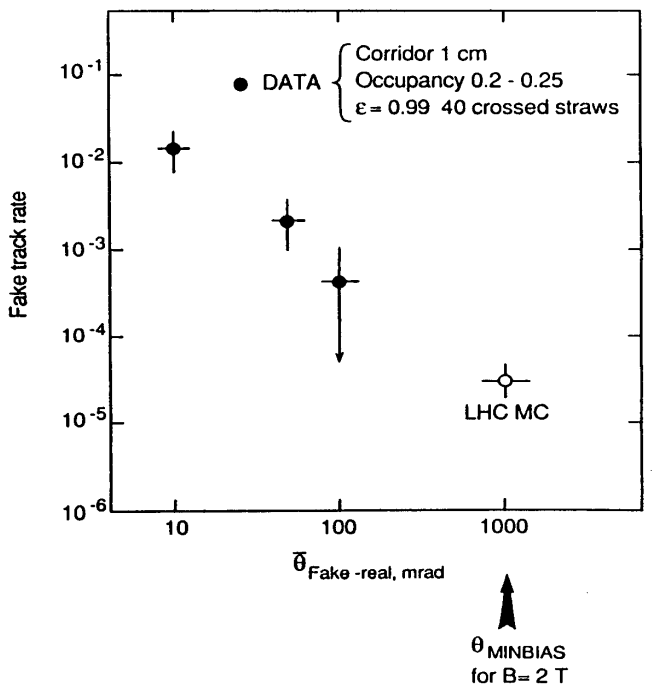

Figure 32: Fake track rate for different degrees of hit correlations using target run data and Monte-Carlo simulations for extrapolations to $L H C$

straws per track remains close to 40 .

All the experimental results presented here have been compared to Monte-Carlo simulations. The satisfactory agreement between the experimental data and the Monte-Carlo simulations gives confidence in the reliability of the Monte Carlo studies of the full-scale TRT performance at the LHC.

\subsection{Backsplash Studies}

In the context of the ATLAS inner detector, one of the problems which may arise when trying to realistically describe the straw occupancy might be the backsplash rate from the electromagnetic calorimeter, which consists of high density material. Although this affects mostly electromagnetically interacting particles $(e, \gamma)$, any significant deviation from the Monte-Carlo prediction may result in underestimating the occupancy in the outermost tracking layers.

The backsplash probability was measured [23] using a lead brick positioned in the magnetic field $\mathrm{B}=0.78 \mathrm{~T}$, about $5 \mathrm{~cm}$ behind the TRT prototype, in order to maximize any backsplash effects. This distance will be at least $40 \mathrm{~cm}$ in the case of ATLAS. Although the transverse component of the magnetic field was about 10 times lower than in ATLAS, a large fraction of the backsplash particles were focused back into the TRT thus maximizing the backsplash effect. Data were taken to study in particular the dependence of the backsplash from the lead brick on the energy of the incoming particle, the particle type $(e, \pi)$, the angle between the particle and the brick surface and the magnetic field. The number and the energy spectrum of hits outside the road of the incoming particles, which consisted mainly of $\delta$-electrons and backsplash particles, were studied and compared to Monte-Carlo predictions.

No discrepancy was observed between the Monte-Carlo and the data in the absence of backsplash. Every feature due to backsplash observed in the experimental data was also reproduced by the GEANT Monte-Carlo simulation. However, in the case of maximum backsplash, i.e. for a brick at $45^{\circ}$, for $\mathrm{B}=0.78 \mathrm{~T}$ and for $60 \mathrm{GeV}$ electrons, twice as many backsplash hits were observed near the lead brick in the data as in the Monte-Carlo prediction, as shown in fig. 33. On the other hand, the experimental energy spectrum of the hits near the brick showed no significant deviation from the simulated spectrum. The total kinetic energy of all backsplash particles crossing the TRT is predicted to be on average only $2.4 \mathrm{MeV}$ for a primary electron of $60 \mathrm{GeV}$, and is expected to be significantly less for the lower energy particles produced in minimum bias events at LHC.

In order to draw conclusions on whether backsplash will contribute significantly to the occupancy of the ATLAS inner detector, the backsplash probability from photons, which are expected to be most abundantly produced at LHC, was also evaluated by extrapolating the Monte-Carlo simulation. The 


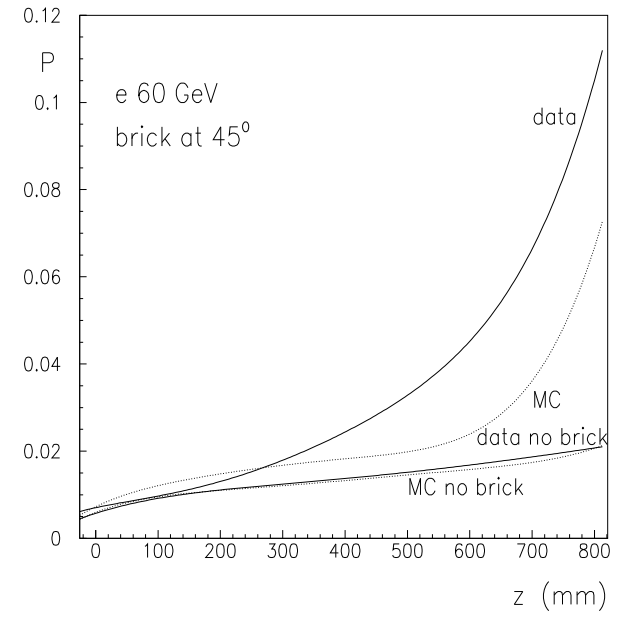

Figure 33: Comparison of the straw hit probability $P$ outside the track road between data (full line) and simulation (dotted line), for $60 \mathrm{GeV}$ electrons with a lead brick at an angle of $45^{\circ}$ in a magnetic field $B=0.78 \mathrm{~T}$. Also shown are the data (full) and simulation (dotted) without backsplash (60 GeV electrons without brick and in the magnetic field). The probability is shown as a function of $z$, the longitudinal position of the straw along the beam (large $z$ corresponds to straws closest to the lead brick).

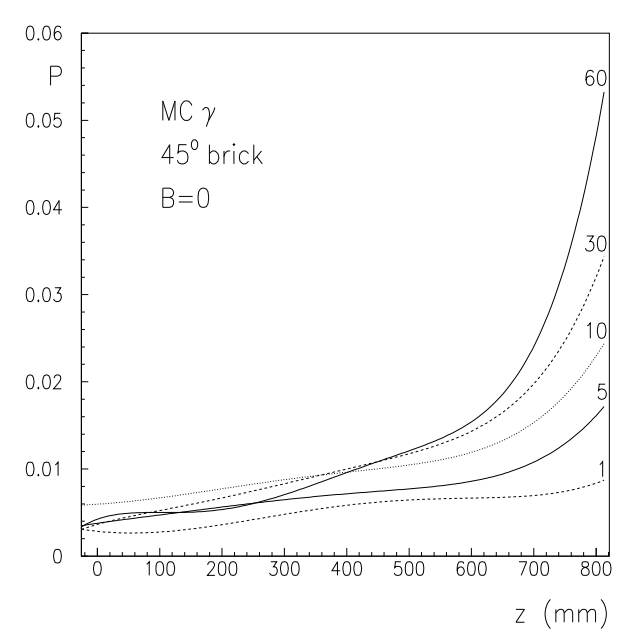

Figure 34: Comparison of the predicted straw hit probability $P$ outside the track road between photons with lead brick at an angle of $45^{\circ}$ in a magnetic field at $60 \mathrm{GeV}$ (full line), $30 \mathrm{GeV}$ (dashed line), $10 \mathrm{GeV}$ (dotted), $5 \mathrm{GeV}$ (full) and $1 \mathrm{GeV}$ (dashed). 
expected backsplash from photons was found to be about $25 \%$ smaller than from electrons. The predicted dependence of the backsplash on the energy of the photons is shown in fig. 34 . It can be seen that below $1 \mathrm{GeV}$ photon energy, the backsplash contribution is negligible.

To summarise, the discrepancy between data and Monte-Carlo, (a factor 2 at most), does not have any serious implications for LHC, since the predicted backsplash occupancy from the low energy minimum bias tracks is much smaller than the occupancy due to the tracks themselves.

\section{Conclusions}

By a series of experimental measurements with a Transition Radiation Tracker prototype and extensive Monte-Carlo studies, it has been shown that such a detector can provide pattern recognition and electron identification in a high multiplicity environment and would therefore be a suitable option as part of the inner tracker of an experiment at the LHC.

For an electron efficiency of $90 \%$ and an average of 40 crossed straws over a prototype length of $80 \mathrm{~cm}$, rejections of $\sim 30$ against $20 \mathrm{GeV}$ pions and $\sim 50$ against photon conversions without magnetic field were measured in an environment corresponding to the expected $\sim 20 \%$ average straw occupancy at LHC.

The fake track rates were measured for an average straw occupancy close to that expected at LHC and found to be between $10^{-2}$ and $5 \times 10^{-4}$, depending on the correlations from straw to straw. By extrapolation to the situation expected in a $2 \mathrm{~T}$ magnetic field at $\mathrm{LHC}$, a fake track rate of $3 \times 10^{-5}$ would be expected at design luminosity.

The good agreement between the experimental data and detailed Monte-Carlo simulations of the TRT prototype, not only corroborates the measurements presented in this paper, but also gives confidence in the reliability of Monte-Carlo predictions of the performance of the TRT in the ATLAS inner detector at LHC.

\section{Acknowledgements}

We are very grateful to the RD3 Collaboration for allowing us to work with the Liquid Argon Accordion Calorimeter, and for their help in adapting their data acquisition system to our needs, and we thank K. Bussmann and G. Di-Tore for their continuous contributions to the preparation of mechanics and electronics for the test beam runs.

The research described in this publication was partly made possible thanks to the following funding agencies: The European Union (DGXII), The International Science Foundation (grant NM5J000), KBNThe Polish State Committee for Scientific Research (grant 115/E-343 SPUB-20693), The Swedish Natural Science Research Council, The Swedish Council for Planning and Coordination of Research, The UK Particle Physics and Astronomy Research Council, and the INTAS 93-57 fund.

\section{References}

[1] T. Åkesson et al., Proceedings of the Workshop on Experiments, Detectors, and Experimental Areas for the Supercollider, July $7 / 17,1987$, Berkeley, California, pages 472/509

[2] T. Åkesson et al., CERN 88/02, 22 April 1988, pages 31/38

[3] V. A. Polychronakos et al., CERN/DRDC/90-38, DRDC/P8

[4] V. A. Polychronakos et al., CERN/DRDC/91-47, RD6/Status Report

[5] J. T. Shank et al., Nucl. Instr. Meth. A309(1991)377/385

[6] R. DeSalvo, Proceedings of the 1986 Summer Study on the Physics of the Superconducting Supercollider, June 23/July 11, 1986, Snowmass, Colorado, page 391 ;

A. Odian, ibid., page 398

[7] M.L. Cherry et al., Phys. Rev. D 10(1974)3594

[8] V. Ermilova et al., Nucl. Instr. Meth. A145(1977)

[9] W.W.M. Allison and J.H. Cobb, Ann. Rev. NUcl. Part. Sci. 30(1980)253

[10] ATLAS Technical Proposal, CERN/LHCC 94-43

[11] V. Commishau et al., CERN/DRDC/93-46, RD6/Status Report

[12] V. Chernyatin et al., Nucl. Instr. Meth. A325(1993)441/445

[13] V. Bondarenko et al., Nucl. Instr. Meth. A327(1993)386

[14] T. Åkesson et al., CERN-PPE/94-224, 15 December 1994, submitted to Nucl. Instr. Meth.

[15] CERN/DRDC/90-31, DRDC/P5, 13 August 1990.

[16] CERN/DRDC/91-11, DRDC/P5-Add.1, 13 March 1991

[17] B. Yu et al., BNL 52244

[18] RD3 Collaboration, CERN-PPE/92-206, 24 November 1993. 
[19] J. Fischer et al., Nucl. Instr. Meth. A238(1985)249/269

[20] Tracking performance of a Straw Transition Radiation Tracker Prototype, submitted to Nucl. Instr. Meth.

[21] P. Nevski, Proc. Int. Europhysics Conf. on High Energy Physics, page 386, Marseille, July 22-28, 1993

[22] RD6 Collaboration, ATLAS internal note INDET-No-18, 29 September 1993

[23] W. Funk, ATLAS internal note INDET-No-32, 16 November 1993 